\title{
Evaluation and Prediction of Evoked Emotions Induced by Image Manipulations
}

\author{
Lin Yuan and Touradj Ebrahimi \\ Multimedia Signal Processing Group, EPFL, 1015 Lausanne, Switzerland
}

\begin{abstract}
Various image editing tools make our pictures more attractive, and at the same time, evoke different emotional responses. With powerful and easy-to-use imaging applications, capturing, editing and then sharing pictures have become daily life for many. This paper investigates the influence of several image manipulations on evoked emotions for different types of images. To do so, various types of images clustered in different categories, were collected from Instagram and subjective evaluations were conducted via crowdsourcing to gather the emotional responses on different manipulations as perceived by subjects. Evaluation results show that certain image manipulations can induce different evoked emotions on transformed pictures when compared to the original ones. However, such changes in image emotions due to manipulation are highly content dependent. Then, we conducted a machine learning based experiment, in attempt to predict the emotions of a manipulated image given its original version and the desired manipulation method. Experimental results present a promising performance of such a prediction model, which could pave the road to automatic selection or recommendation of image editing tools that can efficiently transform or emphasize desired emotions in pictures.
\end{abstract}

\section{Introduction}

Thanks to wide spread popularity of smart mobile devices with high-resolution cameras, as well as user-friendly imaging and social networking applications, taking pictures, then editing and sharing, have become part of everyday life for many. Photo sharing has been used as a way to share not only stories but also current moods with friends, family and public at large. Modern photo sharing applications equipped with advanced and easy-touse image editing tools, such as Instagram, provide consumers with very convenient solutions to make their pictures more attractive, and more importantly, to arouse stronger emotional resonances. Different types of image content generate different emotions. Using different photographic techniques, visual filters or editing tools, pictures of the same scene can also evoke different emotions. Motivated by these facts, we attempt to change an original picture's evoked emotion and transform it to new emotions (stronger, weaker, or completely different) by image manipulation. To achieve this goal, we first need to understand the emotional responses evoked by different image manipulations when applied to pictures.

This paper investigates the influence of image manipulations on evoked emotions, and tries to find the potential pattern between image manipulation and generated emotions. To do so, we conducted subjective experiments based on online crowdsourcing. Different types of images were collected from Instagram, and manipulated by a number of typical image editing tools. Crowdsourcing subjects were then exposed to each, and questioned regarding the emotions pictures induced on them. Using the crowdsourced data as groundtruth, we trained and evaluated a model based on machine learning for predicting evoked emotions, taking an original image and desired manipulation as input.

The rest of the paper is structured as follows. The next section introduces the related works by other researchers, followed by a section describing the data collection and user study. Then we analyze and interpret emotional responses obtained from subjects, and report the experiments of emotion prediction upon image manipulation in the followed two sections. Finally, the last section concludes the paper and discusses future work.

\section{Prior Work}

Image aesthetic quality estimation, emotion recognition and classification have been largely studied in the field of computer vision [1, 2, 3, 4, 5]. Most previous works use image features for affective image classification and emotion prediction [2, 3, 6, 7, 5]. Such features include color, texture, composition, edge and semantic information. A few researchers have worked on transforming image emotions by editing images. In [8], Wang et al. associate color themes with emotion keywords depending on art theory and transform the color theme of an input image to the desired one. However, in their work, only a few cartoon-like images are used. Peng et al. [9] propose a framework to change an image's emotion by randomly sampling from a set of possible target images, but only show a few examples. Jun et al. [10] show that changing brightness and contrast of an image can affect the pleasure and excitement felt by observers. However, only a limited variation of an input image can be produced by changing the two features. Peng et al. [11] change the color tone and texture related features of an image to transfer the evoked emotion distribution, with experiments conducted on only limited types of image content.

Evaluating image's evoked emotions after image manipulation is not a trivial task. Many well-established image manipulation and editing tools have been widely used in online photo sharing and social networks, as ways for users to enhance their image content either to draw better attention or to evoke stronger emotions. Popular image editing tools include image enhancement [12], grayscale conversion, vintage processing, cartoonizing [13], and more recently addition of stickers 1 [14]. However, most image manipulation methods have been studied merely from the perspective of image processing and not so much on their emotional impact.

${ }_{1}^{1}$ https://www .facebook. com/help/1597631423793468 


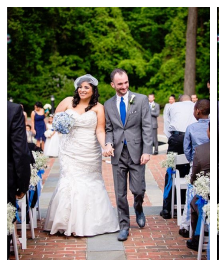

(a) Original

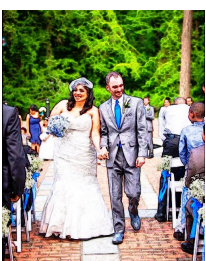

(b) Cartoon

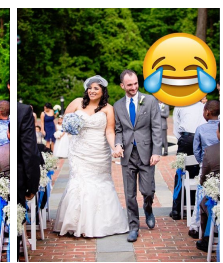

(c) Emoji

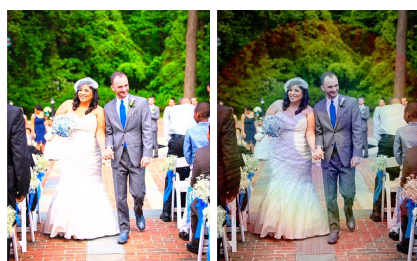

(d) Enhance (e) Halo

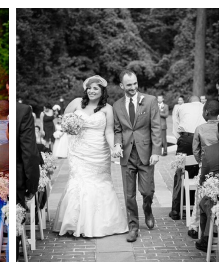

(f) Gray

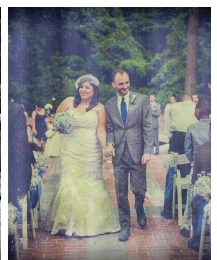

(g) Grunge

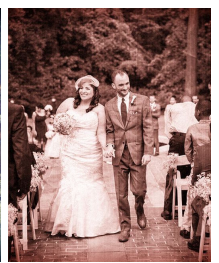

(h) Old paper

Figure 1. Example image manipulated by different methods.

Several affective image databases have been created in previous works, including artistic photos or abstract paintings used in [2], International Affective Picture System (IAPS) [15], The Geneva affective picture database (GAPED) [16] and Emotion6 [11]. In our research, we are more interested in the emotions of everyday photographs, especially those images that are widely shared by online users. Unfortunately, most existing affective image datasets contain either extremely emotional images, or images without much natural high-level semantic features like human face. All those types of images do not fit our requirements. Therefore we decided to collect our own dataset using Instagram, one of the most popular online photo sharing services.

To measure emotions, different types of models have been designed by psychologists. One of the most popular is the valence-arousal (VA) model (proposed by Russell [17]), characterizing emotions in two dimensions, where valence measures attractiveness in a scale from positive to negative, while arousal indicates the degree of excitement or stimulation. In terms of categorization of emotions, Ekman's six basic emotions (anger, disgust, fear, joy, sadness and surprise) [18] are widely known. In our work, we used both models similar to works in [16, 11].

\section{Image Dataset and User Study}

This section describes in detail the image dataset creation and crowdsourcing experiment.

\section{Image Collection and Processing}

We collected images from Instagram. According to a previous study by $\mathrm{Hu}$ et al. [19], images shared within Instagram can be classified into the following eight basic categories in terms of their content: Friends, Food, Gadget, Captioned photo, Pet, Activity, Selfie and Fashion. Therefore, we collected image dataset by searching for the eight category keywords or their synonyms via Instagram \#tag. This was mainly motivated in order to have a wider variety of image content. At the end 13 color images were selected manually for each category resulting in 104 images in total. All selected images have the same size of $640 \times 640$ pixels.

For each image, seven different manipulations were applied to create different visual effects. We will refer to these manipulations as the following names:

- Cartoon: Applies a cartoon effect to an image.

- Emoji: Adds an Emoji on top-right corner of an image.

- Enhance: Applies brightness/contrast/colorization enhancement on an image via LAB colorspace.

- Halo: Applies a circular halo effect to an image.

- Gray: Converts an image to gray scale.
- Grunge: Applies a classic vintage effect with a grunge background to an image.

- Old paper: Applies another heritage style vintage effect with an old paper background to an image.

The reason of selecting the seven particular manipulations is that the changes of an image caused by these operations cover different aspects of image information, e.g. color, texture, composition, and higher-level image semantics. The emoji sticker "Tear of Joy" was selected as it has been in the top 10 most popular emojis on Emojipedia for all of 2015 $5^{2}$ and the emotion it expresses is not that obvious. The seven manipulations were implemented by using ImageMagick software An example image processed by the 7 different manipulations is illustrated in Figure 1 Summing up, a grand total of $832(104 \times 8)$ images were generated, including the original versions of each image. The image dataset is publicly accessible at http://mmspg.epfl.ch/ emotion-image-datasets.

\section{User Study}

We used Microworkers ${ }^{4}$ platform to collect emotional responses from subjects. A questionnaire was designed where four emotion-related questions are asked for each image. The first two questions are about the valence and arousal ratings respectively, where a 9-point scale was used, same as [11, 15]. For valence, 1,5 , and 9 mean very negative, neutral, and very positive emotions respectively, in terms of attractiveness. For arousal, 1 and 9 mean emotions with very low and very high stimulating effects respectively. In the questionnaire, instead of directly asking subjects to provide VA scores, questions were rephrased to be similar as in [11]. The third question is about the emotion distribution of the image, based on Ekman's six basic emotions [18]. Similar to [11], 7 emotion keywords (Ekman's six basic emotions and "Neutral") were used and subjects were asked to select the keywords that best describe their emotions after seeing a particular picture. In the last question, subjects were asked to select the content related factors that have the most impact on their emotional decisions. The 7 pre-defined factors are Face, Color, Scene, Object, Text, Emoji and Halo respectively, and their meanings are shown in http://grebvm2.epfl.ch/lin/emotion/ ImageEmotionEvaluation2.pdf This could further help understanding how the image content and manipulation jointly influence evoked emotions. Furthermore, in every questionnaire, a number of CAPTCHA questions (e.g., " $56+78=$ ?" and "If the

${ }^{2}$ http://emojipedia.org/face-with-tears-of-joy/

3 http://www.imagemagick.org/script/composite.php

4 https://microworkers.com/ 


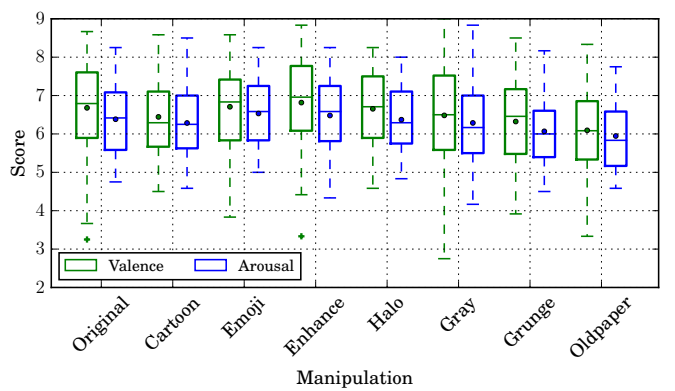

Figure 2. Boxplot of VA scores for each image manipulation method.

arm is green, what color is it?") were included to detect and remove subjects who provided sloppy answers.

Each questionnaire contains eight images, selected in a semirandom way such that the following constraints are satisfied: i) the eight images in each questionnaire came from eight different categories respectively; ii) the order of image manipulations appearing in each questionnaire was randomized. Therefore it ensured that subjects saw different image content in each questionnaire with manipulations in different orders. We aimed at collecting twenty answers for each questionnaire, meaning that every image was to be rated by 20 different answers. Therefore, a total of 2080 $(20 \times 832 / 8)$ questionnaires (implemented using internal template by Microworkers) were distributed online.

Thanks to Microworkers' rating system, we kept tracking the results and ruling out answers from dishonest subjects while task campaigns were running, based on their answers to CAPTCHA questions and the time spent on each questionnaire (for those who spent less than 120 seconds to answer a questionnaire, their answers were removed). The vacated positions were then taken by new subjects until their answers satisfied the above requirement. Finally, answers from 590 unique subjects were collected, each rating 28.2 images in average. We offered 0.3 US dollars to reward the subjects successful completion of each questionnaire. A screenshot of the questionnaire can be found on http://grebvm2.epfl.ch/lin/emotion/ ImageEmotionEvaluation2.pdf

\section{Evaluting Emotions induced by Image Manip- ulation}

This section analyzes the emotional responses obtained from crowdsourcing experiment, with respect to the answers to each question.

\section{Valence-Arousal Score Analysis}

Firstly, for each image, the mean valence and arousal scores were computed, by averaging all the rated VA scores. Then for each image manipulation (including the original), distributions of all images' mean VA scores are gathered and plotted with box plot in Figure 2. Among all the manipulations, vintage processing with Grunge and Old paper generated the lowest VA scores. Besides, for certain methods such as Gray, VA scores show a higher variance than that of other methods. We take as working hypothesis that manipulating an image in a certain way leads evoked emotions to change along a certain direction, but the change of emotions due to image manipulation highly depends on image content.

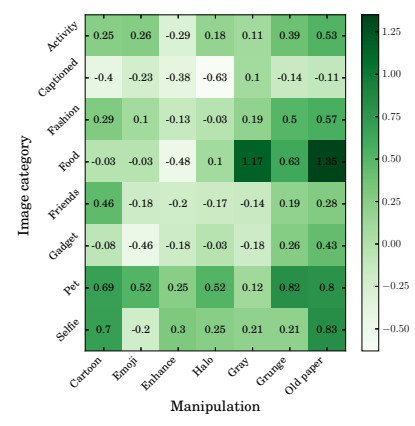

(a) Valence

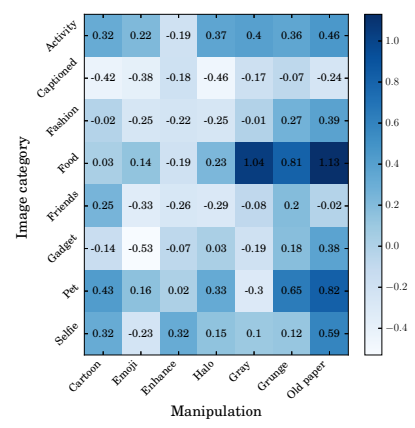

(b) Arousal
Figure 3. Difference VA scores for different image content and manipulations. Value in the map = original score - score after manipulation.

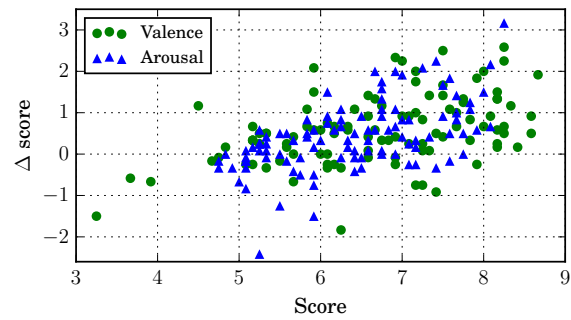

Figure 4. VA score changes due to "Old paper" manipulation, versus original VA scores.

To verify this assumption, we investigated the influence of the two factors (image content and manipulation) on evoked emotion, with respect to VA scores. We computed the mean VA scores of each image category for different manipulation methods, by averaging the scores of 13 pictures belonging to each category for each method. Then we present the difference VA score between original and manipulated images, with respect to different image categories, as heat maps shown in Figure 3 From the results, one observes that certain image manipulations have great impact on the VA scores of certain types of images. For example, manipulations Grunge and Old paper greatly lower the VA scores of most image content, especially for Food and Pet images. In addition, other manipulations like Emoji and Halo both increased the VA scores of Gadget and Captioned photo.

We then plot the $\Delta$ VA scores of every image (difference score between original and manipulated version by Old paper), versus the their original VA scores, as a scatter plot shown in Figure 4 Here, we observe that images with higher original valence or arousal scores are more likely to generate higher difference VA scores, indicating that images with higher VA scores are prone to be impacted by the manipulation Old paper.

\section{Emotion Distribution Analysis}

We then assessed the evoked emotions in terms of probability distribution of emotional keywords. To obtain the emotion distribution of each image, we counted the occurrence of each emotion keyword voted by subjects on each image, and generated a normalized distribution over the 7 keywords, by dividing the number of keyword with the total number of voted keywords. The average emotion distribution corresponding to each manipulation method is shown in Figure 5 Again, one observes slight changes on the 


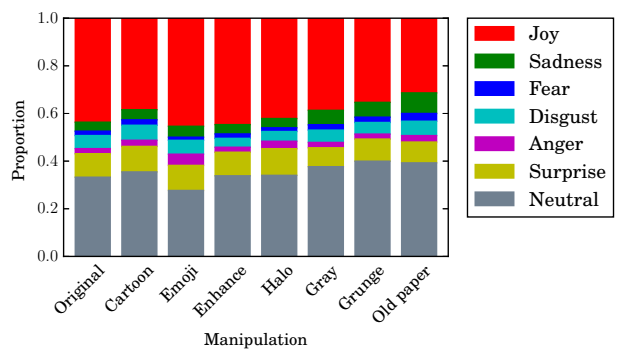

Figure 5. Average emotion distribution of different manipulation methods.

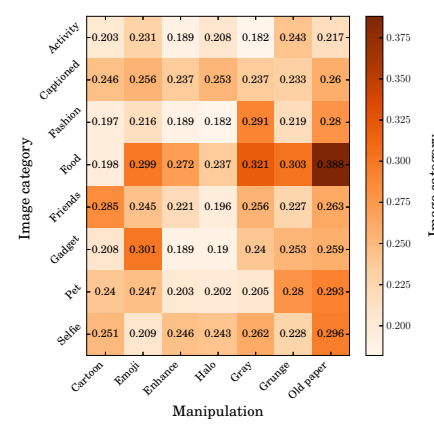

(a) ED

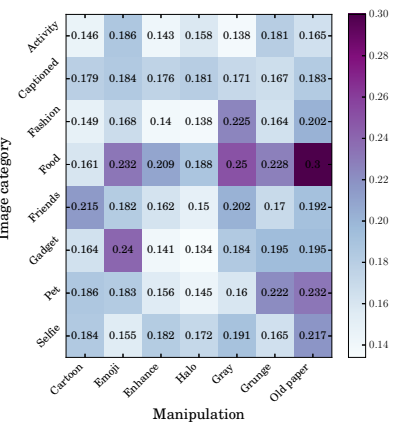

(b) $\mathrm{CD}$
Figure 6. Difference in emotion distributions between manipulated and original images measured by $E D$ and $C D$.

emotion distributions over different manipulation methods. The most obvious observation is that the proportion of Joy for images processed by the two vintage filters (Grunge and Old paper) is much reduced compared to the original images. At the same time, subjects have been evoked more Sadness and Neutral emotions by the two methods.

To quantify the changes in emotion distributions induced by different manipulations, we used two metrics Euclidean distance (ED) and Chebyshev distance (CD) to compute the difference between emotion distributions of original images and their manipulated versions. The average distances for different image categories and manipulations are plotted as heat maps shown in Figure 6 This time, one observes again that different types of image content are influenced by image manipulation methods in different degrees. Similar as previous results on VA scores, methods Gray and Old paper generate higher distances in emotion distributions of many types of images, especially Food and Pet. In some types of images such as Fashion, Food, Gadget and Pet, manipulations on those images have greater impact on evoked emotion distributions than other types of content.

We then plotted the distances (CD) in emotion distributions versus the absolute difference in VA scores, between original images and manipulated images by Old paper, as scatter plot shown in Figure 7 From the result, one observes that when a manipulation has more impact on VA scores, it also tends to create higher differences in emotion distributions. This shows a good degree of correspondence between the emotional changes measured by the two metrics: VA scores and emotion distributions.

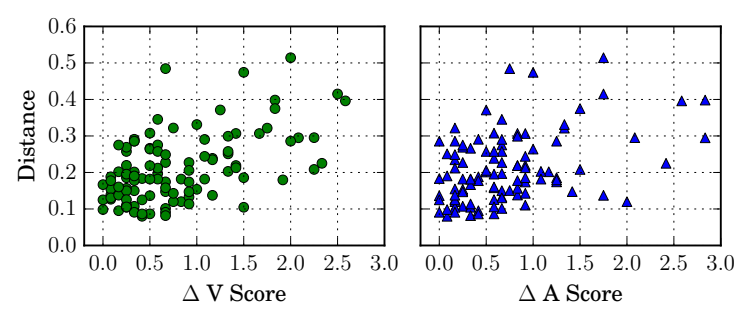

Figure 7. Emotion distribution distance $(C D)$ between original and manipulated image ("Old paper"), versus original VA scores.

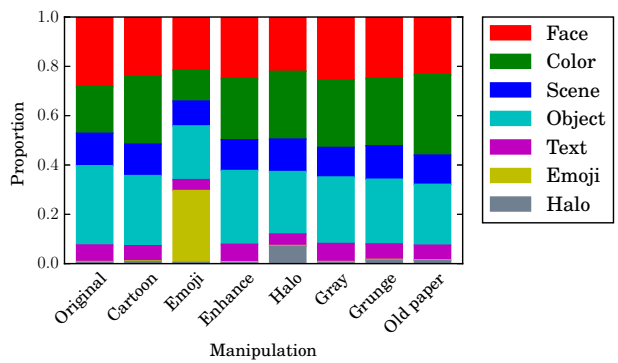

Figure 8. Distribution of influential factors for different manipulations.

\section{Factors Influencing Evoked Emotion}

Furthermore, we investigated the content related factors that influence subjects emotional responses. We computed the probability distributions of subjects selected factors that influenced their decisions, in the same way as computing emotion keyword distribution, and plot the average distribution of each manipulation in Figure 8. In all manipulations, factors like Face, Color and Object highly influence subjects' evoked emotions. However, certain image manipulations that modify image's regional visual information can draw subjects' attentions and facilitate their decision making as well. For instance, in images manipulated by Emoji and Halo, the influential degree of Emoji sticker and halo effect is much increased. In addition, we also notice that the factor Color has a greater influence in most manipulated images (except for Emoji). This is because most manipulations largely change the color information of images, which impacts subjects emotion in a high degree.

\section{Predicting Emotions induced by Image Ma- nipulation}

In this section, we report the experiment of predicting emotions of manipulated images using machine learning. The purpose of conducting this experiment is to investigate the feasibility of accurately predicting image emotions induced by image manipulation before applying the manipulation, and find the potential pattern between image manipulation and evoked emotions.

We propose a predictor which can estimate the emotions of the manipulated version of an image given only the original image and the target manipulation method as input. The targets to be predicted include: the i) valence score, ii) arousal score and iii) emotion distribution. The framework of such a prediction model is illustrated in Figure 9 Such a predictor provides us with at least two benefits. First, it can predict the emotions of desired manipulated image without actually applying any manipulation, which is especially good for computational costly operations, such as 
Table 2. The feature set used for predicting image emotions.

\begin{tabular}{|c|c|l|}
\hline Feature Type & Dimension & Description \\
\hline \multirow{2}{*}{ Color } & 1 & A global factor measuring the colorfulness of a natural image [20]. \\
& 1 & $\begin{array}{l}\text { A global contrast factor of an image [21]. } \\
\text { Color histogram of image YCbCr colorspace (16-bin histogram for each channel). }\end{array}$ \\
\hline Texture & 22 & Features from Gray-Level Co-occurrence Matrix (GLCM) including the mean, variance, energy, entropy, etc [2]. \\
\hline Semantic & 1 & Number of people in the image. \\
\hline Manipulation & 1 & Manipulation method to be applied on the image. \\
\hline
\end{tabular}

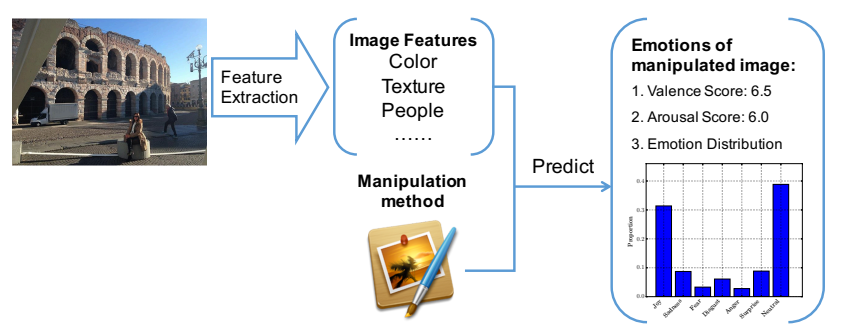

Figure 9. Framework of the emotion prediction system.

the recently popular image editing app Prisme 5 that uses artistic style image morphing. Second, the proposed predictor extracts image features only once from the original image, which does not require the system to extract features each time from newly manipulated images.

To train and evaluate the proposed predictor, we utilized the image dataset used in crowdsourcing experiment. In our dataset, there are 104 images, each manipulated by 7 different methods, resulted in 728 manipulated image samples. To train the predictor, we extracted a set of features (color, texture and semantic information) from each original image, and then took another feature indicating the selected manipulation method. It finally resulted in a 74-dimension feature vector for each image. The detailed descriptions of the features are listed in Table 1

To train and test our models, we used Scikit-learn library [22], within which we experimented with four methods: linear regression (LR), support vector regression (SVR) with Radial basis function (RBF) kernel, random forest regression (RFR), and a baseline method where the predicted value is simply the mean of the training set corresponding to each manipulation method (just for comparison). To avoid over-fitting, we conducted training and testing in 10-fold cross validation, with $90 \%$ images used for training and the rest for testing in each fold. To evaluate the performance of trained predictors, two metrics were used: mean squared error (MSE) and coefficient of determination (denoted $R^{2}$ ). Particularly, for emotion distribution, the MSE value was obtained by averaging the squared differences between predicted distribution and groundtruth distribution over all the 7 dimensions. For each prediction target, we tuned the parameters of each predictor to obtain as good result as we could. The final average results of cross validation tests are shown in Table 2

From the results, one observes that the random forest regression outperforms the other three methods. It resulted in the maximal $R^{2}$ scores higher than 0.5 in predicting all the three targets: 0.639 for valence, 0.571 for arousal and 0.585 for emotion distribution, which are much higher than that of random guessing $\left(R^{2} \leq 0\right)$ or the baseline method. When compared to the results

\footnotetext{
5 http://prisma-ai.com/
}

Table 3. Prediction results of cross validation experiments.

\begin{tabular}{|l|c|c|c|c|c|c|}
\hline \multirow{2}{*}{ Method } & \multicolumn{2}{|c|}{ Valence } & \multicolumn{2}{c|}{ Arousal } & \multicolumn{2}{c|}{ Emotion Distribution } \\
\cline { 2 - 7 } & $M S E$ & $R^{2}$ & $M S E$ & $R^{2}$ & $M S E$ & $R^{2}$ \\
\hline Baseline & 1.21 & $\sim 0$ & 0.75 & $\sim 0$ & 0.0132 & $\sim 0$ \\
LR & 0.78 & 0.369 & 0.57 & 0.311 & 0.0092 & 0.325 \\
SVR & 0.51 & 0.589 & 0.37 & 0.529 & 0.0060 & 0.559 \\
RFR & 0.45 & 0.639 & 0.34 & 0.571 & 0.0056 & 0.585 \\
\hline
\end{tabular}

of VA score prediction in [5], where the minimal MSE of 1.27 for valence and 0.82 for arousal were obtained, our model in prediction of VA scores seems promising, although different datasets were used. With random forest regression, we also checked the importance of features in decision making, and found that the manipulation method, number of people and energy of GLCM are the three most important features. This again indicates that image manipulation indeed influences image emotion in a high degree, and that high-level semantic features like the existence of people also have great impact on evoked emotion, in addition to other low-level image features such as color and texture.

\section{Conclusion}

This paper investigates the influence of image manipulation on evoked emotions for different types of images. An image dataset was created by collecting different types of images from Instagram, and subjective experiments were conducted via online crowdsourcing, to examine subjects emotional responses on different images processed by different manipulation methods. Experimental results show that certain image manipulations induce evoked emotions very different from those experienced on original images. However, such manipulation methods do not always perform the same on different types of images. In other words, emotion changes due to image manipulation are highly dependent on image content. A further experiment based on machine learning was conducted, in attempt to predict the emotions of a manipulated image given only its original version and the manipulation method. Experimental results show a very promising accuracy of using such a model for predicting emotions. These results provide us with insights to design of advanced image emotion transformation systems that can recommend the type of manipulation to apply, based on the content of a picture and the desired emotion to express, which is a future direction of our work.

\section{Acknowledgments}

The research behind this publication was conducted in the framework of Swiss National Foundation for Scientific Research project LEADME (200020-149259). 


\section{References}

[1] D. Joshi, R. Datta, E. Fedorovskaya, Q.-T. Luong, J. Z. Wang, J. Li, and J. Luo, "Aesthetics and emotions in images," Signal Processing Magazine, IEEE, vol. 28, no. 5, pp. 94-115, 2011.

[2] J. Machajdik and A. Hanbury, "Affective image classification using features inspired by psychology and art theory," in Proceedings of the 18th ACM International Conference on Multimedia, ser. MM '10. ACM, 2010, pp. 83-92.

[3] M. Solli and R. Lenz, "Emotion related structures in large image databases," in Proceedings of the ACM International Conference on Image and Video Retrieval, ser. CIVR ' 10. New York, NY, USA: ACM, 2010, pp. 398-405.

[4] X. Wang, J. Jia, J. Yin, and L. Cai, "Interpretable aesthetic features for affective image classification," in Image Processing (ICIP), 2013 20th IEEE International Conference on, Sept 2013, pp. 3230-3234.

[5] S. Zhao, Y. Gao, X. Jiang, H. Yao, T.-S. Chua, and X. Sun, "Exploring principles-of-art features for image emotion recognition," in Proceedings of the 22Nd ACM International Conference on Multimedia, ser. MM '14, pp. 47-56.

[6] Y.-Y. Chen, T. Chen, W. H. Hsu, H.-Y. M. Liao, and S.-F. Chang, "Predicting viewer affective comments based on image content in social media," in Proceedings of International Conference on Multimedia Retrieval, ser. ICMR '14. New York, NY, USA: ACM, 2014, pp. 233:233-233:240.

[7] B. Jou, S. Bhattacharya, and S.-F. Chang, "Predicting viewer perceived emotions in animated GIFs," in Proceedings of the 22nd ACM International Conference on Multimedia, ser. MM '14. New York, NY, USA: ACM, 2014, pp. 213-216.

[8] X. Wang, J. Jia, and L. Cai, "Affective image adjustment with a single word," Vis. Comput., vol. 29, no. 11, pp. 11211133, Nov. 2013.

[9] K.-C. Peng, K. Karlsson, T. Chen, D.-Q. Zhang, and H. Yu, "A framework of changing image emotion using emotion prediction," in Image Processing (ICIP), 2014 IEEE International Conference on, Oct 2014.

[10] J. Jun, L.-C. Ou, B. Oicherman, S.-T. Wei, M. R. Luo, H. Nachilieli, and C. Staelin, "Psychophysical and psychophysiological measurement of image emotion," in Color and Imaging Conference, vol. 2010, no. 1. Society for Imaging Science and Technology, 2010, pp. 121-127.

[11] K.-C. Peng, T. Chen, A. Sadovnik, and A. C. Gallagher, "A mixed bag of emotions: Model, predict, and transfer emotion distributions," in CVPR. IEEE, 2015, pp. 860-868.

[12] J. L. Starck, F. Murtagh, E. J. Candes, and D. L. Donoho, "Gray and color image contrast enhancement by the curvelet transform," IEEE Transactions on Image Processing, vol. 12, no. 6, pp. 706-717, June 2003.

[13] C. Wang, J. Zhang, B. Yang, and L. Zhang, "Sketch2cartoon: Composing cartoon images by sketching," in Proceedings of the 19th ACM International Conference on Multimedia, ser. MM '11, pp. 789-790.

[14] L. Yuan and T. Ebrahimi, "Image transmorphing with JPEG," in 2015 IEEE International Conference on Image Processing (ICIP), Sept 2015, pp. 3956-3960.

[15] P. J. Lang, M. M. Bradley, and B. N. Cuthbert, "International affective picture system (IAPS): Affective ratings of pictures and instruction manual," The Center for Research in Psychophysiology, University of Florida, Gainesville, FL, Tech. Rep. A-8, 2008.

[16] E. S. Dan-Glauser and K. R. Scherer, "The Geneva affective picture database (GAPED): a new 730-picture database focusing on valence and normative significance," Behavior research methods, vol. 43, no. 2, pp. 468-477, 2011.

[17] J. Russell, "A circumplex model of affect," Journal of personality and social psychology, vol. 39, no. 6, pp. 11611178, 1980.

[18] P. Ekman, W. V. Friese, and P. Ellsworth, "CHAPTER XIII - what emotion categories can observers judge from facial behavior?" in Emotion in the Human Face, ser. Pergamon General Psychology Series, P. E. V. F. ELLSWORTH, Ed. Pergamon, 1972, vol. 11, pp. $57-65$.

[19] Y. Hu, L. Manikonda, and S. Kambhampati, "What we instagram: A first analysis of instagram photo content and user types," in Proceedings of the 8th International Conference on Weblogs and Social Media, ICWSM 2014. The AAAI Press, 2014, pp. 595-598.

[20] D. Hasler and S. E. Suesstrunk, "Measuring colorfulness in natural images," in Proc. SPIE, vol. 5007, 2003, pp. 87-95.

[21] K. Matković, L. Neumann, A. Neumann, T. Psik, and W. Purgathofer, "Global contrast factor - a new approach to image contrast," in Proceedings of the First Eurographics Conference on Computational Aesthetics in Graphics, Visualization and Imaging, ser. Computational Aesthetics'05. Aire-la-Ville, Switzerland, Switzerland: Eurographics Association, 2005, pp. 159-167.

[22] F. Pedregosa, G. Varoquaux, A. Gramfort, and et al., "Scikitlearn: Machine learning in Python," Journal of Machine Learning Research, vol. 12, pp. 2825-2830, 2011.

\section{Author Biography}

Lin Yuan received his B.S. and M.Sc. degrees in both Electronic Engineering from University of Electronic Science and Technology of China (UESTC) and Swiss Federal Institute of Technology in Lausanne (EPFL), in 2011 and 2013 respectively. Since then he has been working as a Ph.D. candidate in the Multimedia Signal Processing Group of EPFL, supervised by Prof. Touradj Ebrahimi. His work has been focused on image privacy protection, image context and emotion analysis. He was with Technicolor R\&I in Hannover for one-year internship between 2012-2013.

Touradj Ebrahimi received his M.Sc. and Ph.D. both in Electrical Engineering, from the Swiss Federal Institute of Technology in Lausanne (EPFL), in 1989 and 1992 respectively. He is currently Professor at EPFL heading Multimedia Signal Processing Group. He is also the Convenor of JPEG standardization Committee. He was also adjunct Professor with the Center of Quantifiable Quality of Service at Norwegian University of Science and Technology (NTNU) from 2008 to 2012. In 1993, he was a research engineer at the Corporate Research Laboratories of Sony Corporation in Tokyo, where he conducted research on advanced video compression techniques for storage applications. In 1994, he served as a research consultant at AT\&T Bell Laboratories working on very low bitrate video coding. His research interests include still, moving, and $3 D$ image processing and coding, visual information security, new media, and human computer interfaces. He is a member of IEEE, SPIE, ACM and IS\&T. 